\section{Time for cigarette reclassification?}

SIR - With so much ado about smoking one would think that official classifications of cigarettes would provide a reliable estimate of smoke intake from different brands. Alas, it is not so. Classifications to this day rely on analytical machines that puff cigarettes only at standard volume and frequency under standard laboratory conditions. In reality smokers puff and then inhale in amounts that vary from moment to moment, and draw into the lungs either much more or much less smoke than 'standard' smoking machines.

Today, surely, it is possible to develop an improved cigarette classification, because smoke intake can now be quantified by internalized markers. Of these, carbon monoxide, thiocyanate and others suffer from external interference whereas nicotine and cotinine are suitably specific. Virtually all available studies agree that plasma steady-state levels rise only around 2-4 per cent for nicotine and cotinine, compared with the increase of analytical machine yields measured by the smaller discriminant unit proffered in

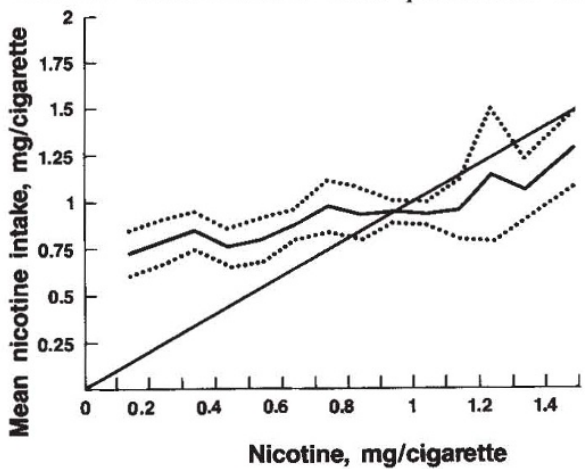

Nicotine intake as a function of analytical cigarette yield. Yields determined by the FTC (Federal Trade Commission) standard method. Dotted lines, 95 per cent confidence intervals. Solid straight line, domain where intake would equal analytical yield (from ref. 5).

statutes and advertising, namely 0.1 milligrams for nicotine ${ }^{1-7}$.

Based on pharmacokinetic considerations, we concluded ${ }^{5}$ that actual nicotine intake per cigarette increases by less than 3 per cent in relation to increasing analytical nicotine yields. Specifically, while such yields increase 1,400 per cent over the range $0.1-1.5$ micrograms per cigarette, the corresponding mean nicotine intake per cigarette increases by only about 40 per cent (see figure).

It is thought that momentary behavioural and pharmacodynamic demand for nicotine modulates puffing and inhalation. At the same time, the observed daily steady-state plasma nicotine levels show that cumulative demand may be limited at quasi-stable ceilings characteristic of each individual. It is conceivable that nicotine would have aversive effects if personal thresholds are exceeded.

Depending on how they are smoked, nearly all cigarettes deliver enough nicotine to meet the fluctuating demands of any smoker, and therefore standard analytical yields of nicotine are meaningless as quantitative predictors of nicotine intake. Of course this is also true of tar yields. On the other hand, for any nicotine inhaled the corresponding amount of tar inhaled is defined by the tar-to-nicotine ratio of the smoke, a characteristic of each brand. A classification based on such ratios would then say what proportion of tar intake the smoker can expect from different brands.

Tar-to-nicotine ratios change little within the range of smoking dynamics, the estimated variance being $\pm 10-15$ per cent ${ }^{8,9}$. This would allow smoking machines to define and rank tarto-nicotine ratios for different brands, especially after improvements in analytical sensitivity. Arguably, such ranking may have to account for the modest increase of intake when analytical yields increase. To this end, I suggest as a suitable formula: $\operatorname{rank}=T(1+0.3 N) / N$,
SIR-John Maddox ${ }^{1}$ has rejected the Big Bang model of the Universe as "philosophically unacceptable" and Jean-Marc Lévy-Leblond" has replied, "it need not be as 'philosophically unacceptable' as he contends." But Maddox expects, and Lévy-Leblond allows, that scientific evidence will turn out to justify the abandonment of the model.

I claim that, insofar as both its classical and quantum versions become unacceptable, they will do so only on scientific, rather than on philosophical grounds. For example, the very recently discovered "great wall" and "great attractor", the so-called "dark matter", the newly observed most distant and oldest quasars ${ }^{3}$ and the role played by plasma in cosmic evolution $^{4}$ pose a theoretical challenge that the Big Bang framework may, in due course, be unable to meet.

But there are basic logical flaws in the assumptions that prompted Maddox's philosophical rejection of the cataclysmic cosmic scenario as well as in LévyLeblond's claim that his linear time has enhanced the conceptual pallatability of the Big Bang. They either beg the question or illicitly impose a priori demands on physical cosmology.

The "philosophical difficulty" that Maddox sees in the Big Bang model is "that an important issue, that of the ultimate origin of our world, cannot be discussed". His argument is predicated on where $T$ and $N$ are mean standard machine yields of tar and nicotine in milligrams per cigarette, and 0.3 is a proportionality factor ${ }^{5}$. A continuous ranking scale of tar-to-nicotine ratios would encourage the development of cigarettes offering progressively lower tar and benign levels of nicotine, a trend already apparent in the market ${ }^{10}$ and officially considered as desirable ${ }^{11}$.

\section{GıO BATTA GORI}

The Health Policy Center,

6704 Barr Road,

Bethesda,

Maryland 20816, USA

1. Hill, P. \& Marquardt, H. Clin. pharmac. Ther. 27, 652$658(1980)$

2. Russell, M.A.H. et al. Br. Med. J. 280, 972-976 (1980).

. Benowitz, N.L. et al. New Engl. I. Med. 309, 139-142 (1983)

4. Ebert, R.V. et al. J. Am. med. Ass. 250, 2940-2942 (1983).

. Gori, G.B. \& Lynch, C.J. Reg. Tox. Pharmac. 5, 314-326 (1985)

6. Russell, M.A.H. et al. J. epidem. Comm. Health $\mathbf{4 0}$ 80-85 (1986)

7. Benowitz, N.L. et al. J. Am. med. Ass. 256, 241-246 (1986).

8. Browne, C.L., Keith, C.H. \& Allen, R.E. Beitrage zur Tabakforschung Internationa/ 10, 81-90 (1980).

9. Schlotzhauer, W.S. \& Chortyk, O.T. J. analyt. Toxicol. 7 92-95 (1983).

10. Jarvis, M. \& Russell, M.A.H. Br. J. Addict. 81, 579-581 (1986).

11. Waild, N. \& Frogatt, P. (eds) Nicotine, Smoking and the Low Tar Programme (Oxford University Press. 1989).

\title{
Pseudo-creation of the Big Bang
}

the occurrence of the cosmic cataclysm at a "well-defined instant" $t_{0}$ which had no temporal predecessor. But as Torretti has explained ${ }^{5}$, general relativity theory does not countenance $t_{0}$ as a bona fide instant of the Big Bang space-time.

Yet the alleged philosophical difficulty would be spurious, I contend, even if the model did feature a time-interval that is closed, rather than open, for $t=t_{0}$. By hypothesis, there simply did not exist any instants before it. But precisely this total absence of times earlier than $t_{0}$ also rules out the very existence of an earlier cause of any event that does occur at the hypothesized instant $t_{0}$. Hence, if the Big Bang is taken to have occurred at the putative $t_{0}$, that initial event is causally sui generis. It just cannot have any cause at all in the universe of the given model, nor, of course, can that paramount occurrence be the effect of any prior cause.

Therefore, it just begs the question to insist, as Maddox does peremptorily, on characterizing the supposedly initial event as "an effect". By thus illicitly requiring the existence of an earlier cause within the assumed model, this characterization also requires the existence of at least one instant before $t_{0}$ after all, which saddles the cosmological model with a temporal inconsistency. Maddox objects that, qua purported effect, the Big Bang "is an effect whose cause cannot be identified or even discussed", but the elusiveness of the 